\title{
Endoplasmic reticulum oxidoreductase 1 alpha modulates prostate cancer hallmarks
}

\author{
Julian Cornelius $^{1,2}$, Ilaria Cavarretta ${ }^{1}$, Edoardo Pozzi ${ }^{1,3}$, Giovanni Lavorgna ${ }^{1}$, Irene Locatelli ${ }^{1}$, \\ Tiziana Tempio ${ }^{4}$, Francesco Montorsi ${ }^{1,3}$, Agostino Mattei ${ }^{2}$, Roberto Sitia ${ }^{3,4}$, Andrea Salonia ${ }^{1,3}$, \\ Tiziana Anelli ${ }^{3,4}$
}

${ }^{1}$ Division of Experimental Oncology/Unit of Urology, URI, IRCCS Ospedale San Raffaele, Milan, Italy; ${ }^{2}$ Department of Urology, Luzerner Kantonsspital, Lucerne, Switzerland; ${ }^{3}$ University Vita-Salute San Raffaele, Milan, Italy; ${ }^{4}$ Division of Genetics and Cell Biology, IRCCS Ospedale San Raffaele, Milan, Italy

Contributions: (I) Concept and design: T Anelli, I Cavarretta, J Cornelius, A Salonia, F Montorsi, A Mattei, R Sitia; (II) Administrative support: I Cavarretta, T Anelli, I Locatelli, F Montorsi, A Mattei, R Sitia, A Salonia; (III) Provision of study materials or patients: I Cavarretta, T Anelli, A Salonia, R Sitia; (IV) Collection and assembly of data: J Cornelius, I Cavarretta, E Pozzi, T Tempio, G Lavorgna, T Anelli; (V) Data analysis and interpretation: J Cornelius, I Cavarretta, E Pozzi, R Sitia, T Anelli; (VI) Manuscript writing: All authors; (VII) Final approval of manuscript: All authors.

Correspondence to: Julian Cornelius. Department of Urology, Luzerner Kantonsspital, Lucerne, Switzerland. Email: julian.cornelius@luks.ch.

Background: Therapies available for late stage prostate cancer (PCa) patients are limited and mostly palliative. The necessary development of unexplored therapeutic options relies on a deeper knowledge of molecular mechanisms leading to cancer progression. Redox signals are known to modulate the intensity and duration of oncogenic circuits; cues originating from the endoplasmic reticulum (ER) and downstream exocytic organelles are relevant in secretory tumors, including PCa. Ero $1 \alpha$ is a master regulator of redox homeostasis and oxidative folding.

Methods: We assessed Ero $1 \alpha$ mRNA expression by bioinformatic analysis of three public datasets and protein expression levels in PCa cell lines representing different degrees of tumor progression and different human prostate specimens. Transient Ero $1 \alpha$ knockdown was achieved by RNA interference (siRNA). Consequences of Ero $1 \alpha$ downregulation were monitored by PCa proliferation, migration and invasion properties.

Results: Ero $1 \alpha \mathrm{mRNA}$ and protein levels are upregulated in PCa cell lines compared to non-tumorigenic cells $(\mathrm{P}=0.0273)$. Ero $1 \alpha$ expression increases with the grade of malignancy, reaching the highest level in the androgen resistant PC3. In patients' samples from 3 datasets, Ero $1 \alpha$ mRNA expression correlates with pathological Gleason scores. Ero $1 \alpha$ knockdown inhibits proliferation $(\mathrm{P}=0.0081)$, migration $(\mathrm{P}=0.0085)$ and invasion $(\mathrm{P}=0.0007)$ of $\mathrm{PC} 3$ cells and alters the levels of integrin $\beta 1(\mathrm{P}=0.0024)$.

Conclusions: Results indicate that Ero $1 \alpha$ levels correlate with PCa aggressiveness; Ero $1 \alpha$ silencing inhibits key steps over the PCa metastatic process. Therefore, Ero $1 \alpha$ has the potential to be exploited as a novel biomarker and a therapeutic target in $\mathrm{PCa}$.

Keywords: Prostate cancer (PCa); endoplasmatic reticulum; endoplasmic reticulum oxidoreductase 1 alpha (Ero $1 \alpha)$; redoxstasis; Gleason score

Submitted Jun 21, 2020. Accepted for publication Nov 15, 2020.

doi: $10.21037 /$ tau-20-1025

View this article at: http://dx.doi.org/10.21037/tau-20-1025 


\section{Introduction}

Tumor progression is sustained by aberrant signaling circuits that are largely dependent on redox-dependent pathways. Signals originating from the endoplasmic reticulum (ER) and downstream exocytic organelles have particular relevance in prostate cancer $(\mathrm{PCa})$ and other secretory tumors $(1,2)$. The biosynthesis of secretory proteins often requires disulfide bond formation (2). The processes of 'oxidative folding' set in motion electron transport chains that ultimately generate $\mathrm{H}_{2} \mathrm{O}_{2}$ (3). ER oxidoreductin 1 alpha (Ero $1 \alpha$ ) flavoproteins are rate-limiting steps in oxidative folding. They oxidize protein disulphide-isomerase (PDI), which in turn donates disulphide bonds to nascent proteins $(4,5)$. Ero $1 \alpha$ is upregulated under conditions of hypoxia (6) and ER stress (7), thus generating $\mathrm{H}_{2} \mathrm{O}_{2}$ that can be either transported to the cytosol or utilized lumenally by peroxiredoxin 4 (PRDX4) (8) or glutathione peroxidases (GPX) 7 and 8 (9). As a consequence, the relative amount of these molecules determine redox homeostasis in the ER and $\mathrm{H}_{2} \mathrm{O}_{2}$ fluxes. $\mathrm{H}_{2} \mathrm{O}_{2}$ can amplify oncogenic signals, inhibiting protein tyrosine phosphatases. Conversely, if redundant, $\mathrm{H}_{2} \mathrm{O}_{2}$ can induce lethal oxidative stress (10). Ero $1 \alpha$ can also modulate cell life-death decisions activating inositol 1,4, 5 - triphosphate receptors (11).

Tumorigenesis and cancer progression are very complex processes during which cells acquire different peculiar characteristics $(12,13)$. In particular, hypoxia is a frequent condition during tumorigenesis and a key feature of the tumor microenvironment (14). Ero $1 \alpha$ has been proposed as a marker of hypoxia in various kinds of cancers as it is induced by low-oxygenic environments via the hypoxiainducible factor 1 (HIF-1) (15). It also controls mRNA levels and folding of vascular endothelial growth factor A (VEGF-A), a molecule essential for neoangiogenesis (16). Furthermore, Ero $1 \alpha$ promotes neoangiogenesis and metastasis by targeting the S1PR1/STAT3/VEGF-A pathway in hepatocellular carcinoma (17).

With an increased rate of protein assembly and folding within the ER (to ensure efficient protein supply), cancer cells are often exposed to a higher risk for misfolded protein accumulation with consequent -stress and induction of Unfolded Protein Response (UPR) (6); this has profound impact on tumor progression, neo-angiogenesis, tumor invasion and metastasis (18). While Ero $1 \alpha$ is predominantly induced under hypoxia, the isoform Ero $1 \beta$ is mainly induced under UPR (6).

Ero $1 \alpha$ plays also a key role in the regulation of immune response to cancer. It has been reported to control MHC class I folding and expression in colorectal cancer cells, by altering the susceptibility of tumorigenic cells by $\mathrm{CD} 8^{+} \mathrm{T}$ cells (19). Furthermore, a link between Ero $1 \alpha$ mediated oxidative folding and programmed death-ligand 1 (PD-L1) expression levels has been observed in breast cancer cells. PD-L1, by binding to programmed death-1 (PD-1) expressed on immune cells, plays a crucial role in tumor immunology. Importantly, Ero $1 \alpha$ levels have been found correlated to PD-L1 expression in vitro and in vivo, suggesting Ero $1 \alpha$ as a potential target in cancer immunotherapy (20).

Consistently with these evidences, Ero $1 \alpha$ has been found correlated with bad prognosis in different types of cancers, such as breast, pancreatic, cervical and gastric cancers (21-24).

The deregulation/activation of the described pathways and mechanisms is common to many malignancies including PCa. Based on these evidences, we hypothesize that Ero $1 \alpha$ may play a relevant role in PCa progression too.

We investigated (I) Ero $1 \alpha$ expression in human tumoral and not tumoral prostate cell lines and prostate samples; and, (II) Ero $1 \alpha$ role in modulating PCa cell proliferative signaling, migration ability, and invasive potential.

We present the following article in accordance with the MDAR reporting checklist (available at http://dx.doi. org/10.21037/tau-20-1025).

\section{Methods}

\section{Reagents and prostate cell lines}

All reagents and chemicals were from Thermo Fisher Scientific Inc. (Waltham, Massachusetts, USA), unless otherwise stated. The human epithelial prostate cell line RWPE-1 (RRID: CVCL_3792) was purchased from ATCC and maintained in Keratinocyte Serum Free Medium supplemented with $0.05 \mathrm{mg} / \mathrm{mL}$ bovine pituitary extract and $5 \mathrm{ng} / \mathrm{mL}$ human epidermal growth factor. The human cell lines LNCaP, LAPC4, DU145 and PC3 (respectively, RRID: CVCL_0395, CVCL_4744, CVCL_0105, CVCL_0035) were kindly donated by Prof. Zoran Culig (Innsbruck Medical University, Innsbruck, Austria). LNCaP and PC3 cells were cultured in Roswell Park Memorial Institute 1640 medium supplemented with $10 \%$ fetal calf serum (EuroClone S.p.A., Pero, Milan, Italy). LAPC4 were cultured in Iscove`s Modified Dulbecco`s Medium (IMDM) containing 10\% fetal bovine serum and $100 \mathrm{nM}$ dihydrotestosterone. All culture media contained $100 \mathrm{u} / \mathrm{mL}$ penicillin and $100 \mu \mathrm{g} / \mathrm{mL}$ streptomycin. 
Docetaxel resistance was obtained by exposing PC3 cells to $5 \mathrm{ng} / \mathrm{mL}$ docetaxel for 1 day followed by gradual removal of the drug. Treatment was repeated every 21 days for 3 times.

\section{Primary patient samples}

Patient samples were collected from patients undergoing either holmium laser enucleation of the prostate (HoLEP) for symptomatic benign prostatic hyperplasia (BPH) or radical prostatectomy $(\mathrm{RP})$ for $\mathrm{PCa}$ at a single tertiaryreferral academic institute. Median age was 63 years (range, 53-73 years). Data collection followed the principles outlined in the Declaration of Helsinki; all patients had signed an informed consent agreeing to deliver their own anonymous information for future studies. The study was approved by our local ethical committee.

\section{siRNA transfection}

Sequences of small interfering RNA (siRNA) inhibiting Ero $1 \alpha$ gene expression (Ambion ${ }^{\circledR}$, Waltham, Massachusetts, USA) were previously published $(25,26)$. Transfection was carried out using Invitrogen ${ }^{\mathrm{TM}}$ Lipofectamine $^{\mathrm{TM}}$ RNAiMAX according to the manufacturer's instructions. Transient protein expression knockdown was confirmed by Western blotting analysis with specific antibodies at 72 hours after silencing.

\section{Protein extraction and western blotting analysis}

Cells were detached from culture flasks or culture plates using $0.25 \%$ Trypsin-EDTA, washed in PBS $10 \mathrm{mM}$ N-ethylmaleimide (NEM) to block disulphide interchange (4). Cells were lysed in RIPA lysis buffer $(150 \mathrm{mM} \mathrm{NaCl}, 1 \% \mathrm{NP}-$ 40, 0.1\% SDS, $50 \mathrm{mM}$ Tris- $\mathrm{HCl} \mathrm{pH} 8.0$ supplemented with $10 \mathrm{mM} \mathrm{NEM}$, and protease inhibitor cocktail) for 20 minutes on ice. Clearance was achieved by centrifugation with $17,000 \times \mathrm{g}$ at $4{ }^{\circ} \mathrm{C}$ for 20 minutes. Post-nuclear supernatants were used immediately or stored at $-20{ }^{\circ} \mathrm{C}$. Human tissue samples, after lysis, were subjected to $30^{\prime \prime}$ sonication (1 pulse/ second; $50 \%$ power/Sonopuls Ultrasonic Homogenizer, Bandelin Electronic GmbH \& Co. Berlin, Germany) followed by centrifugation at $13,000 \mathrm{rpm} / 15^{\prime} / 4^{\circ} \mathrm{C}$. Proteins were quantified by bicinchoninic acid (BCA) assay (Sigma, St Louis, MO, USA).

Proteins were analyzed by Western blotting (reducing SDS-PAGE on gradient 4-12\% Bis- Tris polyacrylamide precasted gels) (Invitrogen ${ }^{\mathrm{TM}}$, Thermo Fisher Scientific).
After electro-transfer to Nitrocellulose paper, nitrocellulose was saturated with $5 \%$ milk in PBS $0.1 \%$ Tween. Nitrocellulose papers were incubated with primary antibodies: mouse monoclonal anti-Ero $1 \alpha$ [2G4, 1:1,000 (27)], anti- $\beta 1$ Integrin (mouse monoclonal clone E4/C9 kind gift of Dr. Maria Raffaella Zocchi (28), rabbit anti-anti PDI 1:1,000 (kind gift from Prof. I. Braakman, Utrecht, The Netherlands); Rabbit anti-GRP94 1:500 (ADI-SPA-851, lot \#08021036, RRID AB_10615790, ENZO Life Sciences, Farmingdale, New York, USA); Rabbit anti-Peroxiredoxin 4 1:1,000 (10703-1-AP, RRID AB_2168493, Invitrogen Proteintech ${ }^{\mathrm{TM}}$, Thermo Fisher Scientific); mouse anti-GAPDH (6C5) 1:2,000 (sc32233, lot \#I0319, RRID AB_627679, Santa Cruz, Dallas, Texas, USA) and mouse monoclonal anti- $\beta$ actin 1:2,000 (A5441, lot \#127M4866v, RRID AB_476744, Sigma, St Louis, MO, USA). Subsequently nitrocellulose membranes were incubated with anti-Mouse IgG or anti-Rabbit Ig secondary antibodies Alexa Fluor conjugated (700, 647 or 488) (Invitrogen ${ }^{\mathrm{TM}}$, Thermo Fisher Scientific) 1:2,000 in PBS $0.1 \%$ Tween (Alexa Fluor ${ }^{\mathrm{TM}} 488$ Goat anti-Mouse IgG $(\mathrm{H}+\mathrm{L})$ A11029, lot \#2066710, RRID AB_138404; Alexa Fluor ${ }^{\text {TM }} 647$ Goat anti-Mouse IgG $(\mathrm{H}+\mathrm{L})$ A21236, lot \#2170302, RRID AB_2535805; Alexa Fluor ${ }^{\mathrm{TM}} 700$ Goat anti-Mouse IgG $(\mathrm{H}+\mathrm{L})$ A21036, lot \#1906489, RRID AB_1500658; Alexa Fluor ${ }^{\text {TM }}$ 488 Goat anti-Rabbit IgG (H+L) A11034, lot \#1851447, RRID AB_2576217; Alexa Fluor ${ }^{\mathrm{TM}} 647$ Goat anti-Rabbit IgG (H+L) A21245, lot \#2098544, RRID AB_2535813; Alexa Fluor ${ }^{\mathrm{TM}} 700$ Goat anti-Rabbit IgG $(\mathrm{H}+\mathrm{L})$ A2 1038, lot \#1875540, RRID AB_1500674). Signals were detected by infrared technology by FujiFilm (FLA 9000) (FujiFilm Life Science, Tokyo, Japan) and densitometric analysis of the images was performed with ImageJ.

\section{Proliferation assay}

A total of 200,000 cells per well were plated on 6-wellplates and treated with siRNA targeting Ero $1 \alpha$ using Lipofectamine ${ }^{\mathrm{TM}}$ RNAiMAX or scrambled siRNA as a control. Forty-eight hours after silencing, cells were plated in $100 \mu \mathrm{L}$ of standard cell-culture medium on a 96-well ImageLock ${ }^{\mathrm{TM}}$ plate (Essen Bioscience, Ann Arbor, Michigan, USA) to reach a confluence of 10-20\% and placed in the IncuCyte S3 Life Cell Analysis System (Essen Bioscience). Images were taken every 2 hours for a total duration of 48 hours.

\section{Migration assay}

In order to analyze the effects of Ero $1 \alpha$ silencing on cell 
migration, 48 hours after Ero $1 \alpha$ siRNA transfection, cells were transferred into a Culture-Insert 2 Well $\mu$-dish (Ibidi, Planegg, Germany) (70,000 cells for chamber). Cells were incubated overnight to allow complete adhesion. The silicon inlay was removed and life-cell microscopy was carried out for up to 24 hours, in RPMI culture medium supplemented with $2 \%$ FCS, with GFP-Imaging (Widefield Imaging Setup) Zeiss Axiovert S100 with Hamamatsu OrcaII-ER. The gap size was measured with Fiji image processing software.

\section{Scratch wound cell invasion assay}

In order to analyze the effect of a downregulation of Ero $1 \alpha$ on PCa cell invasion, we investigated the ability of Ero $1 \alpha$-silenced PC3 cells to invade into a Matrigel Matrix in vitro. Matrigel (Corning) was diluted to final concentration of $100 \mu \mathrm{g} / \mathrm{mL}$ in RPMI culture medium supplemented with $2 \%$ FCS. Wells of a 96-well ImageLock plate were coated with $50 \mu \mathrm{L}$ of diluted Matrigel to create a thin layer and incubated at $37{ }^{\circ} \mathrm{C}$ over-night. Forty-eight hours after silencing of Ero 1 $\alpha$, PC3 cells were seeded at 50,000 cells/ well. Scratch wounds were created using the IncuCyte WoundMaker. A Matrigel top layer was created with $50 \mu \mathrm{L}$ Matrigel solution per well $(2.6 \mathrm{mg} / \mathrm{mL}$ in RPMI $2 \%$ FCS). After $30 \mathrm{~min}$ of incubation at $37^{\circ} \mathrm{C}, 100 \mu \mathrm{L}$ of RPMI culture medium containing $2 \%$ FCS was added to each well and the assay plate was placed into the IncuCyte S3 Life Cell Analysis System. Images were taken every 2 hours for a total of 90 hours.

\section{Bioinformatic analysis}

The Pearson correlation coefficient, along with its significance (P value), between Ero $1 \alpha$ mRNA expression and the Gleason score, was calculated in three PCa datasets (29) by using the R software (https://www.r-project.org/). As for Taylor and TCGA datasets, normalized expression data and clinical data were taken, respectively, from the cBioPortal (http://www.cbioportal.org/study/clinicalData?id=prad_ mskcc) and the Genomic Data Commons Data Portal (https://portal.gdc.cancer.gov/). As for Ross-Adams dataset, normalized expression data and clinical data were obtained from the Gene Expression Omnibus (GEO, GSE70768).

\section{Statistical analysis}

All data are expressed as mean \pm standard deviation
(SD). Welch's $t$-test, Mann-Whitney test and T-test with significance defined as $\mathrm{P}<0.05$ were used for analysis. Graphs were fitted by non-linear regression. To test normality Shapiro-Wilk Test was addressed. Statistical analysis was carried out with Prism GraphPad Version 7.0 for Mac, GraphPad Software, La Jolla California USA, www.graphpad.com".

\section{Ethical statement}

The study was conducted in accordance with the Declaration of Helsinki (as revised 2013). This study was approved by the institutional research committee CEIRCCS San Raffaele (Registration-ID: URBAN) and informed consent was taken from all the patients.

\section{Results}

\section{Ero $1 \alpha$ expression correlates with PCa malignancy}

Dataset analysis of three different, public patient cohorts allowed us to uncover a consistent and significant, positive correlation between Ero $1 \alpha$ mRNA levels and the Gleason patterns of either biopsies and surgical specimens from RP (Table 1). In one cohort a marginal difference in Ero $1 \alpha$ mRNA expression was observed also between primary tumors and metastases (data not shown). Finally, also Ero $1 \alpha$ protein expression, measured by western blot on human prostate samples, revealed a trend to higher levels in PCa samples compared to non-tumoral prostate tissues and $\mathrm{BPH}$ samples (Figure 1A,B).

The same dataset also showed that the more malignant PCa cell line has higher levels of Ero $1 \alpha$ mRNA compared to the less malignant $\mathrm{LNCaP}$ cell line (Table 2). Consistently with these findings, we found differences also at the protein level between PCa cell lines (i.e., LNCaP, LAPC4, Du145 and PC3 cells) and the non-tumorigenic RWPE1 cell line, which was adopted as a control (Figure 1C,D). Again, the highest levels were observed in the more aggressive, androgen-independent, Du145 and PC3. Ero $1 \alpha$ levels further increased in PC3 after achievement of docetaxel resistance (Figure 1E,F). Interestingly, Ero $1 \alpha /$ PRDX4 ratio was higher in the androgen independent cells (i.e., DU145 and PC3) and further increased after achievement of docetaxel resistance (data not shown). Taken together, current findings suggested that Ero $1 \alpha$ levels (both mRNA and protein) correlate both with PCa onset and progression. 
Table 1 Correlation of Ero1 $\alpha$ mRNA expression to Gleason score in three different patient cohorts (19-21)

\begin{tabular}{llll}
\hline Variable & $\mathrm{R}$ & $\mathrm{P}$ & Study \\
\hline gleason_pattern_primary & 0.09 & $4.18 \mathrm{E}-02$ & TCGA [2015] \\
BxGG1 & 0.28 & $1.28 \mathrm{E}-03$ & Taylor et al. [2010] \\
BxGGS & 0.20 & $2.29 \mathrm{E}-02$ & Taylor et al. [2010] \\
PathGGS & 0.22 & $1.34 \mathrm{E}-02$ & Taylor et al. [2010] \\
Primary_Gleason & 0.24 & $6.50 \mathrm{E}-03$ & Ross-Adams et al. [2015] \\
Secondary_Gleason & 0.21 & $2.00 \mathrm{E}-02$ & Ross-Adams et al. [2015] \\
Gleason & 0.36 & $4.59 \mathrm{E}-05$ & Ross-Adams et al. [2015] \\
\hline
\end{tabular}

Ero $1 \alpha$, endoplasmic reticulum oxidoreductin 1 alpha; BxGG1, biopsy primary Gleason grade; BxGGS, biopsy combined Gleason score; PathGGS, combined Gleason score in the radical prostatectomy specimen; R, correlation (Pearson score); $p$, probability.

\section{Ero 1 a knockdown inbibits PCa cell proliferative, migratory and invasive abilities}

We asked whether increased Ero $1 \alpha$ levels are needed to sustain PCa progression. In this context, we examined the effects of Ero $1 \alpha$ downregulation on the proliferation of PC3 and RWPE-1 (Figure 2A,B, respectively). As expected, Ero $1 \alpha$ silencing inhibited the proliferation of PC3 $(\mathrm{P}=0.0081)$. A much lower inhibitory effect was observed in the non-tumorigenic RWPE-1 cells ( $\mathrm{P}=0.2523)$. The extent of Ero $1 \alpha$ silencing was demonstrated by western blot analysis (Figure 2C).

Next, we analyzed the effect of Ero $1 \alpha$ silencing on the migratory properties of PC3 cells. Clearly, cells with lower Ero $1 \alpha$ levels needed significantly longer time to occupy an empty space as compared with untreated cells (Figure $3 A, B$ ). Single cell tracking assays revealed that silenced cells moved with a significantly slower velocity than controls (Figure 3C,D). This observation confirmed that the slower migration shown in panels $\mathrm{A}$ and $\mathrm{B}$ was not the mere consequence of a lower proliferation of Ero $1 \alpha$ silenced cells.

Next step was to investigate whether Ero $1 \alpha$ downregulation affected only cell migration or may also impact on the cellular ability to invade a matrix. Thereof, we compared the ability of PC3 cells to migrate into a dense Matrige ${ }^{\circledR}$ matrix after Ero $1 \alpha$ or Control siRNA transfection (Figure $3 E$ ). To this end, cells grown in a semisolid medium are asked to invade a denser matrix for 90 hours. Clearly, Ero $1 \alpha$ knockdown significantly impaired the migratory capacity of PC3 cells (Figure 3F, $\mathrm{P}=0.0007$ ).

\section{Ero 1a affects integrin $\beta 1$ levels in PC3 cells}

In line with the above data, the vast majority of PC3 control cells displayed the stellate appearance typical of invading cells. In contrast, Ero $1 \alpha$ silenced cells tended to maintain a roundish shape, thus suggesting difficulties in creating the extrusions necessary for cell mobility and invasion (Figure 4A). A possible reason underlying the impairment of migratoryinvasive properties of silenced cells is a defective processing of adhesion molecules. Integrins are adhesion molecules rich in disulfide bonds. Therefore, a decrease of Ero $1 \alpha$ levels could eventually impact their folding and transport. To verify this possibility, we quantified the expression of the adhesion surface molecule, integrin $\beta 1$, in PC3 cells and PC 3 cells silenced for Ero $1 \alpha$ expression. Clearly, downregulation of Ero $1 \alpha$ cells caused a statistically significant decrease of the total amount of integrin 1 (Figure 4B,C).

\section{Discussion}

Tumor progression is a multifactorial process, where mutations and aberrant signals concur to increasing cancer cell 'fitness' in a given microenvironment. In this scenario, reactive oxygen species (ROS) play key hormetic roles particularly in secretory tumors (31). Several fundamental and intertwined aspects link ER redoxstasis to tumor progression: (I) efficient oxidative folding is crucial in terms of production control of angiogenic or adhesion molecules acting inter-cellularly; (II) $\mathrm{H}_{2} \mathrm{O}_{2}$ generated in the ER could potentiate tyrosine receptor signaling; and, (III) the secreted oxidase QSOX favors redox interchange in matrix proteins, and in turn metastasis (32-34).

Due to its essential activity in ensuring efficient oxidative folding (whilst by-producing $\mathrm{H}_{2} \mathrm{O}_{2}$ ) and due to the secretory nature of prostate epithelial cells, we investigated whether and how Ero $1 \alpha$ may affect PCa progression. An 

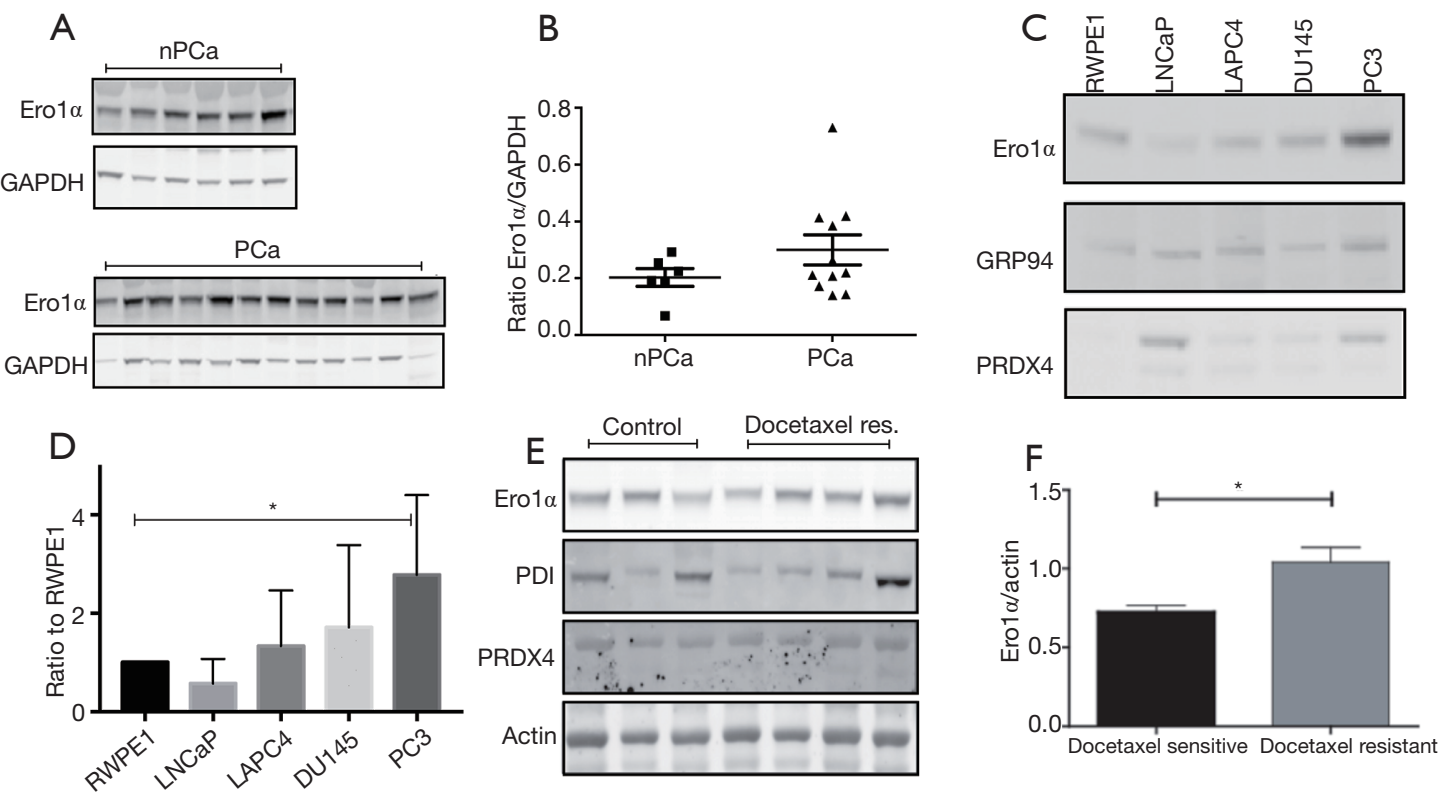

Figure 1 Ero $1 \alpha$ levels are increased in primary patient samples and PCa cell lines. (A) Representative Western blot images of Ero $1 \alpha$ in non- $\mathrm{PCa}(\mathrm{nPCa})$ and $\mathrm{PCa}$ primary patient samples. nPCa include the non tumoral area from $\mathrm{PCa}$ as well as $\mathrm{BPH}$ surgical resections. $40 \mu \mathrm{g}$ of total proteins extracts per lane were loaded on SDS-PAGE under reducing conditions. Nitrocellulose was decorated with anti-Ero $1 \alpha$ and anti-GAPDH antibodies, as indicated. (B) Densitometric quantification of Ero $1 \alpha$ western blots shown in (A) (P=0.1371) (6 and 12 biological replicates for $\mathrm{nPCa}$ and $\mathrm{PCa}$ samples, respectively). (C) Representative Western blot images of Ero $1 \alpha$, Endoplasmin-94 kDa glucose-regulated protein (GRP94) and peroxiredoxin 4 (PRDX4) in prostate cell lines. $40 \mu \mathrm{g}$ of proteins/lane were loaded on SDS-PAGE under reducing conditions. Nitrocellulose was decorated with anti-Ero 1 $\alpha$, anti-GRP94 and anti-PRDX4 antibodies, as indicated. Despite the difference in Ero $1 \alpha$ levels, the protein level of the ER chaperone GRP94 is almost constant, hinting against a general enlargement of the ER. PRX4 is overexpressed in LNCaP and PC3, as described in literature (30). (D) Densitometric quantification of the signal. Mean \pm standard deviation (SD); $\mathrm{n}=4$ (biological replicates). Ero $1 \alpha$ protein level is significantly increased in the highly malignant and metastatic PC3 cells line compared to the non-tumorigenic RWPE1 cell line $(\mathrm{P}=0.0273)$. (E) Representative Western blot images of Ero 1 $\alpha$, Protein Disulphide Isomerase (PDI), PRDX4 and actin in PC3 cells. The expression of Ero $1 \alpha$ is significantly increased in PC3 cells resistant to docetaxel with respect to control PC3 cells (i.e., PC3 cells sensitive to docetaxel) $(\mathrm{P}=0.0192)$, while PDI (as marker of the ER) remains constant, indicating that Ero $1 \alpha$ increase is not a consequence of a general ER enlargement. Lysates of three control PC3 cell samples and four Docetaxel-resistant PC3 cell samples were loaded under reducing conditions on SDS-PAGE and decorated with anti- Ero $1 \alpha$, PDI, PRDX4 and actin antibodies, as indicated. Densitometric analysis of the gel is shown in panel (F). Ero $1 \alpha$ levels were normalized to actin. Mean \pm SD is shown (3 and 4 biological replicates for control and Docetaxel resistant cells respectively). *, level of significance. Ero $1 \alpha$, endoplasmic reticulum oxidoreductin 1 alpha; PCa, prostate cancer.

Table 2 Quantification of Erol $\alpha$ mRNA expression in prostate cancer cell lines from Taylor et al. [2010]

\begin{tabular}{lc}
\hline Cell line & Ero1 $\alpha$ mRNA (normalized expression values) \\
\hline LNCaP & 334 \\
VCap & 692.6 \\
LNCap104R & 713.9 \\
LNCap104S & $1,143.7$ \\
DU145 & $1,956.3$ \\
PC3 & $2,933.9$ \\
\hline
\end{tabular}

Ero $1 \alpha$, endoplasmic reticulum oxidoreductin 1 alpha. 

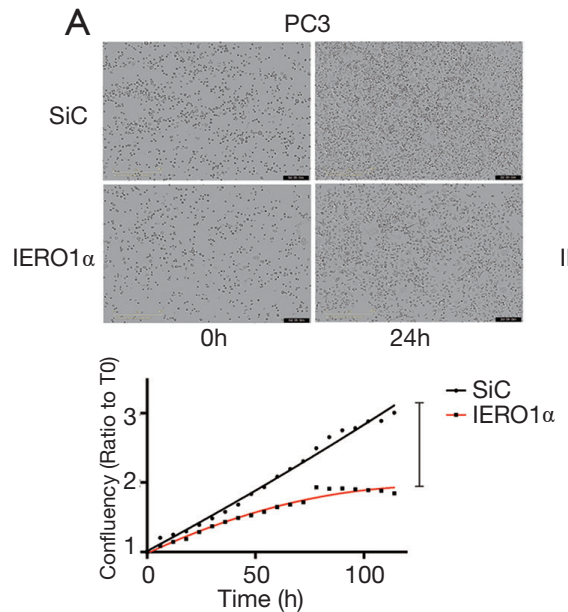
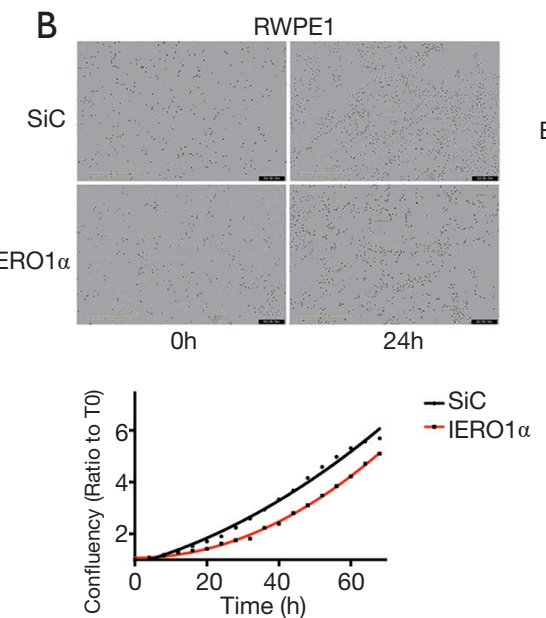

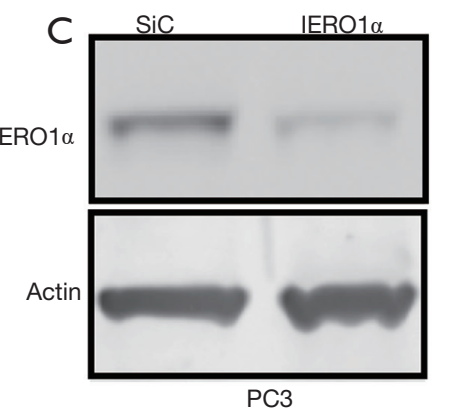

Figure 2 Ero $1 \alpha$ downregulation impairs PCa cell proliferation. (A,B) Representative Fields of View (FOVs) of PC3 and RWPE-1 at time point T0 (0 hours) and T1 (48 hours) during cell proliferation assay. Comparison between cells treated with siRNA Control (siC) or siRNA Ero $1 \alpha$ (iEro 1 $\alpha$ ) shows that cell proliferation rate is significantly inhibited under transient Ero $1 \alpha$ knockdown in the PC3 cells $(\mathrm{P}=0.0081)(\mathrm{A})$ but not in the non-tumorigenic RWPE-1 cells $(\mathrm{P}=0.2532)$ (B). Mean $\pm \mathrm{SD} ; \mathrm{n}=4$ (biological replicates) (C) Western Blot confirming transient protein expression knockdown in PC3 cells. Protein extracts from 100,000 cells/lane were loaded under reducing conditions on SDS-Page and decorated with anti-Ero $1 \alpha$ and anti-actin antibodies. Ero 1 $\alpha$, endoplasmic reticulum oxidoreductin 1 alpha; PCa, prostate cancer.

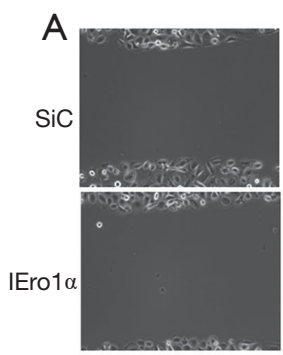

Oh

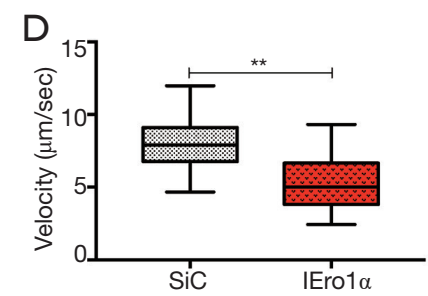

B
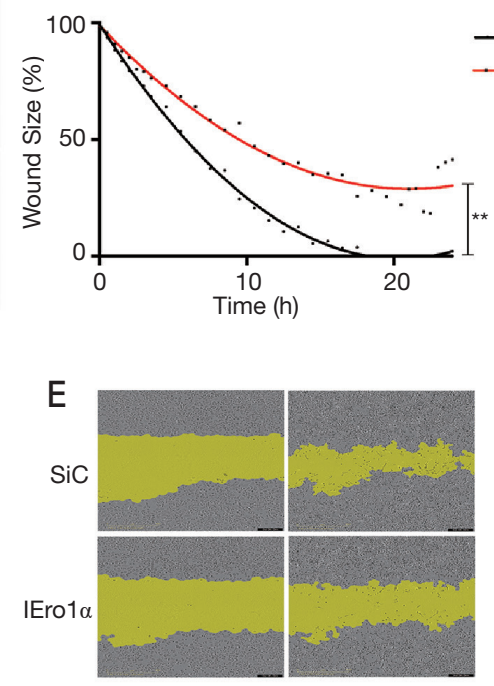

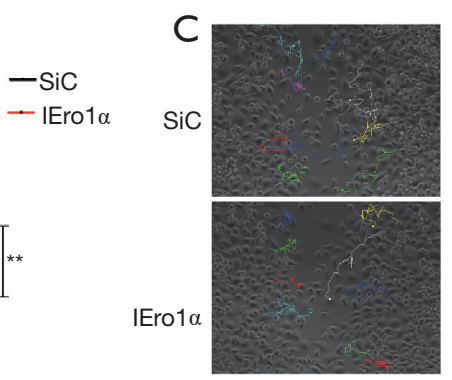

$\mathrm{F}$

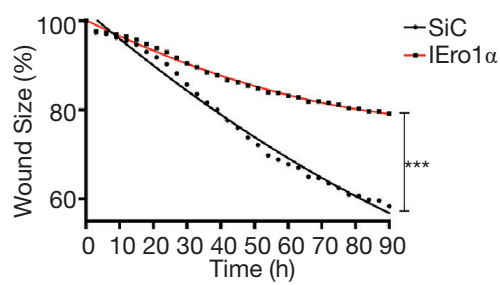

Figure 3 Ero $1 \alpha$ downregulation impairs PCa cell migration and matrix invasion (Migration Assay and Matrigel ${ }^{\circledR}$ Invasion Assay; Incucyte $^{\circledR}$ ). Migration: (A) representative FOVs of migrating PC3 cells at $0 \mathrm{~h}$ and 18 hours after transient transfection with siC or iEro $1 \alpha$. (B) Cell migration is significantly impaired under transient Ero $1 \alpha$ transcription knockdown (siRNA) ( $\mathrm{P}=0.0085)$. Mean $\pm \mathrm{SD} ; \mathrm{n}=3$ (biological replicates). Magnification $\times 100$. Average migration velocity $(\mu \mathrm{m} / \mathrm{s})$ : (C) representative FOVs of migrating PC3 cells, silenced (iEro $1 \alpha$ ) or not (siC) for the expression of Ero $1 \alpha$, manually tracked using Fiji ${ }^{\circledR}$ image processing software. Magnification $\times 100$. (D) Cell migration velocity is significantly lower under transient Ero $1 \alpha(1.002 \pm 0.169)$ transcription knockdown compared to control (siC) (1.985 \pm 0.294$)(\mathrm{P}=0.0061)$. Mean of 10 cells/field from 2 independent experiments (biological replicates). Invasion: (E) invasion was evaluated with the Incucyte ${ }^{\circledR}$ Live-Cell analysis system which provides a real-time quantitative live-cell imaging and analysis. Representative FOVs of PC3 cells invading Matrigel ${ }^{\circledR}$ matrix. Pictures captured after 0 and 90 hours. (F) Cell invasion ability is significantly inhibited by Ero $1 \alpha$ siRNA ( $\mathrm{P}=0.0007)$. After 90 hours, wound size is $58.4 \%$ for $s i C$ and $79.2 \%$ for iEro $1 \alpha$ respect to the initial wound size. Mean $\pm \mathrm{SD} ; \mathrm{n}=4$ (biological replicates). ${ }^{* *},{ }^{* *}$ : level of significance. Ero $1 \alpha$, endoplasmic reticulum oxidoreductin 1 alpha; PCa, prostate cancer. 


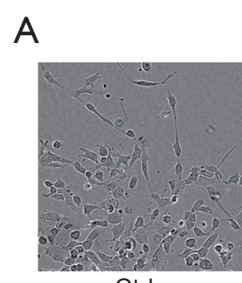

Ctrl

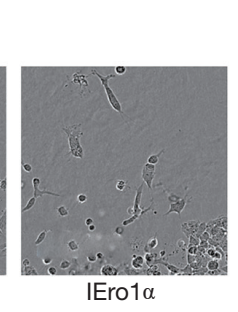

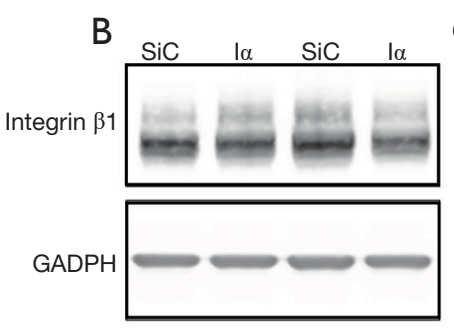

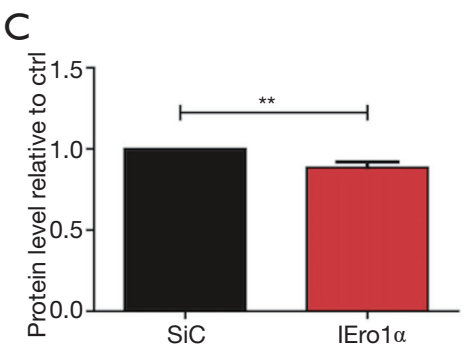

Figure 4 Decreased intracellular levels of Ero $1 \alpha$ affect the morphology of PC 3 cells and the expression of Integrin $\beta 1$. (A) Brightfield microscopy. Transient protein expression knockdown of Ero $1 \alpha$ by siRNA (iEro $1 \alpha$ ) seems to impair formation of cell extensions. Left panel: typical satellite shape of PC3 cells transiently transfected with control siRNA (siC) invading the Matrigel ${ }^{\circledR}$ matrix. Right panel: roundish shape of PC3 cells under transient Ero $1 \alpha$ knockdown (iEro $1 \alpha$ ). Magnification $\times 100$. (B) Representative western blot images of integrin $\beta 1$ and GAPDH in PC3 cells. Densitometric quantification of the gel is shown in panel (C). Levels are normalized to the GAPDH. The expression of Integrin $\beta 1$ is significantly lower after transient knockdown of Ero $1 \alpha$ (i $\alpha$ ) compared to the expression of integrin $\beta 1$ in control cells (siC) $(\mathrm{P}=0.0024)$. Mean $\pm \mathrm{SD} ; \mathrm{n}=12$ (biological replicates). **, level of significance. Ero $1 \alpha$, endoplasmic reticulum oxidoreductin 1 alpha.

analysis of public datasets highlighted a positive correlation between Ero $1 \alpha$ expression and Gleason scores. Although weak, this correlation is consistently maintained in three different patients cohorts (35), both in terms of clinical and pathological scores. Since the Gleason grading system is directly related to a number of histopathological endpoints [including lymph-node vascular space invasion and risk of extra-prostatic extension and metastasis (36)], the observed positive correlation with Ero $1 \alpha$ acquires clinical relevance and can suggest a role over disease progression, as already recently suggested for pancreatic cancer (37) and other secretory malignancies (38).

Consistently, we observed increased Ero $1 \alpha$ protein expression in human surgical PCa specimens compared to control (i.e., the non-tumoral and BPH specimens). The limited number of samples analyzed so far likely accounts for the lack of a significant difference among groups. Further prospective sample collections are needed to clarify this issue. Additionally, among a panel of PCa cell lines, we found higher Ero 1 $\alpha$ mRNA levels in the more malignant, androgen-independent cell lines (i.e., PC3 and Du145) compared to the less malignant, androgen-dependent LNCaP cells. Protein levels matched mRNA quantifications, with PC3 cells having the greatest Ero $1 \alpha$ protein contents. Interestingly, Ero 1 $\alpha$ levels further increased in PC3 cells after establishment of docetaxel resistance. To this regard, it must be noted the link existing between the ER and the androgen/androgen receptor (AR) signaling during $\mathrm{PCa}$ progression. Moreover, various cytoprotective ER chaperons are known to be upregulated by AR stimulation; finally, the expression levels of AR and ER stress genes have been found co-regulated in PCa samples (39). These data encourage investigations on the role of Ero $1 \alpha$ as a pathogenic component and prognostic marker in PCa too.

Cancer's common traits are increased proliferation, migration and invasion, unleashed from the normal control checkpoints (12); our findings revealed a role of Ero $1 \alpha$ in every above mentioned hallmark. Of relevance, Ero $1 \alpha$ suppression had a stronger effect on the highly metastatic PC3 compared to the non-tumorigenic RWPE-1 cells. Consistently, Ero $1 \alpha$ overexpression is reported to promote cell growth $(23,37,40,41)$ and subsequent cell migration and invasion. These properties could be associated to the role of Ero $1 \alpha$ in oxidative protein folding. Moreover, an impaired Ero $1 \alpha$ activity might decrease the production of proteins necessary for efficient migration, invasion, and neoangiogenesis, as the case of the decreased cellsurface integrin- $\beta 1$ expression, consequent to Ero $1 \alpha \mathrm{KO}$ observed in colon cancer cells $(40,42)$. Integrin- $\beta 1$ activation mediates adhesion to the extracellular matrix, thus eventually impacting toward cancer cell migratory and metastatic capacity. This is consistent with our results showing impaired migratory-invasive properties of Ero $1 \alpha$-silenced PC3 cells accompanied by decreased expression of integrin- $\beta 1$. Accordingly, Ero $1 \alpha$ overexpression has been found linked to integrin- $\beta 1$ activation (43).

Overall, with the dramatic results obtained upon silencing, our data provide a rationale for studying/considering Ero $1 \alpha$ as a novel target in advanced PCa therapies. This is also supported by some evidences recently reported in literature. In particular, Ero $1 \alpha$ has been found able to control PD-L1 expression (20) and block the PD1/PD-L1 pathway, thus 
being potentially effective in PCa treatment (44). Since antiPD-1/PDL-1 therapies are explored in PCa too, targeting Ero $1 \alpha$ might allow to refine PCa immunotherapies $(45,46)$.

Again, in hepatocellular carcinoma, Ero $1 \alpha$ stimulates the STAT3 pathway (17), which has a crucial role in PCa metastatic progression too (47). The hypothesis of considering Ero $1 \alpha$ as a therapeutic target in $\mathrm{PCa}$ is further supported by the robust correlations between Ero $1 \alpha$ and relapse or metastatic phenotypes in other malignancies $(23,37)$. Interestingly, a specific targeting of Ero $1 \alpha$ is already under investigation in multiple myeloma (41): the viability of the Ero $1 \alpha$ knockout mice (40) indicate in fact that this has the potential to be a druggable molecule.

However, to clarify the basic question of sensitivity, specificity and prognostic validity, investigations of a large cohorts of healthy patients as well as PCa patients should follow.

\section{Conclusions}

Our novel findings clearly showed that Ero $1 \alpha$ levels are correlated with PCa progression. Of translational importance, selective Ero $1 \alpha$ downregulation inhibited PCa cell proliferation, migration and invasive capacity. As a whole, these observations make Ero $1 \alpha$ a potential prognostic marker and a target for therapeutic strategies and should be further explored.

\section{Acknowledgments}

The authors thank all members of Sitia's laboratory and of URI-Urological Research Institute at IRCCS San Raffaele Hospital, Milan (IT) for their help and stimulating discussions; likewise, ALEMBIC Staff for precious help with the IncuCyte data analysis.

Funding: This work was supported by grants from Telethon (GGP15059), Associazione Italiana per la Ricerca sul Cancro (AIRC, IG 14559) and Fondazione Cariplo (2015-0591) to RS. JC and EP were generously supported by fellowships from the Department of Urology, Luzerner Kantonsspital, Lucerne (CH) and URI, respectively. Additionally, JC was kindly supported by the European Urological Scholarship Programme (EUSP) of the European Association of Urology (EAU).

\section{Footnote}

Reporting Checklist: The authors have completed the MDAR reporting checklist. Available at http://dx.doi.org/10.21037/ tau-20-1025

Data Sharing Statement: available at http://dx.doi. org/10.21037/tau-20-1025

Conflicts of Interest: All authors have completed the ICMJE uniform disclosure form (available at http://dx.doi. org/10.21037/tau-20-1025). Dr. RS reports grants from Telethon (GGP15059), grants from Associazione Italiana per la Ricerca sul Cancro (AIRC, IG 14559) and grants from Fondazione Cariplo (2015-0591), during the conduct of the study. The other authors have no conflict of interests to declare.

Ethical Statement: The authors are accountable for all aspects of the work in ensuring that questions related to the accuracy or integrity of any part of the work are appropriately investigated and resolved. The study was conducted in accordance with the Declaration of Helsinki (as revised 2013). This study was approved by the institutional research committee CE-IRCCS San Raffaele (RegistrationID: URBAN) and informed consent was taken from all the patients.

Open Access Statement: This is an Open Access article distributed in accordance with the Creative Commons Attribution-NonCommercial-NoDerivs 4.0 International License (CC BY-NC-ND 4.0), which permits the noncommercial replication and distribution of the article with the strict proviso that no changes or edits are made and the original work is properly cited (including links to both the formal publication through the relevant DOI and the license). See: https://creativecommons.org/licenses/by-nc-nd/4.0/.

\section{References}

1. Oakes SA. Endoplasmic reticulum proteostasis: a key checkpoint in cancer. Am J Physiol Cell Physiol 2017;312:C93-102.

2. Galmiche A, Sauzay C, Chevet E, et al. Role of the unfolded protein response in tumor cell characteristics and cancer outcome. Curr Opin Oncol 2017;29:41-7.

3. Appenzeller-Herzog C, Riemer J, Christensen B, et al. A novel disulphide switch mechanism in Erolalpha balances ER oxidation in human cells. EMBO J 2008;27:2977-87.

4. Mezghrani A, Fassio A, Benham A, et al. Manipulation of oxidative protein folding and PDI redox state in 
mammalian cells. EMBO J 2001;20:6288-96.

5. Benham AM, Cabibbo A, Fassio A, et al. The CXXCXXC motif determines the folding, structure and stability of human Ero1-La. EMBO J 2000;19:4493-502.

6. Gess B, Hofbauer KH, Wenger RH, et al. The cellular oxygen tension regulates expression of the endoplasmic oxidoreductase ERO1-Lalpha. Eur J Biochem 2003;270:2228-35.

7. Rao J, Zhang C, Wang P, et al. C/EBP homologous protein $(\mathrm{CHOP})$ contributes to hepatocyte death via the promotion of ERO1alpha signalling in acute liver failure. Biochem J 2015;466:369-78.

8. Rhee SG, Woo HA, Kang D. The Role of Peroxiredoxins in the Transduction of H2O2 Signals. Antioxid Redox Signal 2018;28:537-57.

9. Brigelius-Flohé R, Maiorino M. Glutathione peroxidases. Biochim Biophys Acta 2013;1830:3289-303.

10. Medraño-Fernandez I, Bestetti S, Bertolotti M, et al. Stress Regulates Aquaporin-8 Permeability to Impact Cell Growth and Survival. Antioxid Redox Signal 2016;24:1031-44.

11. Li G, Mongillo M, Chin KT, et al. Role of ERO1-alphamediated stimulation of inositol 1,4,5-triphosphate receptor activity in endoplasmic reticulum stress-induced apoptosis. J Cell Biol 2009;186:783-92.

12. Hanahan D, Weinberg RA. The hallmarks of cancer. Cell 2000;100:57-70.

13. Hanahan D, Weinberg RA. Hallmarks of cancer: the next generation. Cell 2011;144:646-74.

14. Clarke HJ, Chambers JE, Liniker E, et al. Endoplasmic reticulum stress in malignancy. Cancer Cell 2014;25:563-73.

15. Takei N, Yoneda A, Kosaka M, et al. ERO1 $\alpha$ is a novel endogenous marker of hypoxia in human cancer cell lines. BMC Cancer 2019;19:510.

16. May D, Itin A, Gal O, et al. Ero1-L alpha plays a key role in a HIF-1-mediated pathway to improve disulfide bond formation and VEGF secretion under hypoxia: implication for cancer. Oncogene 2005;24:1011-20.

17. Yang S, Yang C, Yu F, et al. Endoplasmic reticulum resident oxidase ERO1-Lalpha promotes hepatocellular carcinoma metastasis and angiogenesis through the S1PR1/STAT3/VEGF-A pathway. Cell Death Dis 2018;9:1105.

18. Madden E, Logue SE, Healy SJ, et al. The role of the unfolded protein response in cancer progression: From oncogenesis to chemoresistance. Biol Cell 2019;111:1-17.

19. Kukita K, Tamura Y, Tanaka T, et al. Cancer-Associated
Oxidase ERO1- $\alpha$ Regulates the Expression of MHC Class I Molecule via Oxidative Folding. J Immunol 2015;194:4988-96.

20. Tanaka T, Kutomi G, Kajiwara T, et al. Cancer-associated oxidoreductase ERO1- $\alpha$ promotes immune escape through up-regulation of PD-L1 in human breast cancer. Oncotarget 2017;8:24706-18.

21. Gupta N, Park JE, Tse W, et al. ERO1 $\alpha$ promotes hypoxic tumor progression and is associated with poor prognosis in pancreatic cancer. Oncotarget 2019;10:5970-82.

22. Zhang Y, Li T, Zhang L, et al. Targeting the functional interplay between endoplasmic reticulum oxidoreductin-10 and protein disulfide isomerase suppresses the progression of cervical cancer. EBioMedicine 2019;41:408-19.

23. Kutomi G, Tamura Y, Tanaka T, et al. Human endoplasmic reticulum oxidoreductin 1-alpha is a novel predictor for poor prognosis of breast cancer. Cancer Sci 2013;104:1091-6.

24. Takei N, Yoneda A, Kosaka M, et al. ERO1alpha is a novel endogenous marker of hypoxia in human cancer cell lines. BMC Cancer 2019;19:510.

25. Anelli T, Ceppi S, Bergamelli L, et al. Sequential steps and checkpoints in the early exocytic compartment during secretory IgM biogenesis. EMBO J 2007;26:4177-88.

26. Anelli T, Bergamelli L, Margittai E, et al. Erolalpha regulates $\mathrm{Ca}(2+)$ fluxes at the endoplasmic reticulummitochondria interface (MAM). Antioxid Redox Signal 2012;16:1077-87.

27. Ronzoni R, Anelli T, Brunati M, et al. Pathogenesis of ER storage disorders: modulating Russell body biogenesis by altering proximal and distal quality control. Traffic 2010;11:947-57.

28. Bartolazzi A, Kaczmarek J, Nicolo G, et al. Localization of the alpha 3 beta 1 integrin in some common epithelial tumors of the ovary and in normal equivalents. Anticancer Res 1993;13:1-11.

29. Taylor BS, Schultz N, Hieronymus H, et al. Integrative genomic profiling of human prostate cancer. Cancer Cell 2010;18:11-22.

30. Whitaker HC, Patel D, Howat WJ, et al. Peroxiredoxin-3 is overexpressed in prostate cancer and promotes cancer cell survival by protecting cells from oxidative stress. Br J Cancer 2013;109:983-93.

31. Cao SS, Kaufman RJ. Endoplasmic reticulum stress and oxidative stress in cell fate decision and human disease. Antioxid Redox Signal 2014;21:396-413.

32. Yoboue ED, Sitia R, Simmen T. Redox crosstalk at endoplasmic reticulum (ER) membrane contact sites 
(MCS) uses toxic waste to deliver messages. Cell Death Dis 2018;9:331.

33. Ilani T, Alon A, Grossman I, et al. A secreted disulfide catalyst controls extracellular matrix composition and function. Science 2013;341:74-6.

34. Delaunay-Moisan A, Appenzeller-Herzog C. The antioxidant machinery of the endoplasmic reticulum: Protection and signaling. Free Radic Biol Med 2015;83:341-51.

35. Lamb AD, Bryant RJ, Camilleri P, et al. Orient Expression: Solving the Mystery of Asian Prostate Cancer? Eur Urol 2018;73:340-2.

36. Humphrey PA. Gleason grading and prognostic factors in carcinoma of the prostate. Mod Pathol 2004;17:292-306.

37. Han F, Xu Q, Zhao J, et al. ERO1L promotes pancreatic cancer cell progression through activating the $\mathrm{Wnt} /$ catenin pathway. J Cell Biochem 2018;119:8996-9005.

38. Zhou B, Wang G, Gao S, et al. Expression of ERO1L in gastric cancer and its association with patient prognosis. Exp Ther Med 2017;14:2298-302.

39. Storm M, Sheng X, Arnoldussen YJ, et al. Prostate cancer and the unfolded protein response. Oncotarget 2016;7:54051-66.

40. Takei N, Yoneda A, Sakai-Sawada K, et al. Hypoxiainducible ERO1alpha promotes cancer progression

Cite this article as: Cornelius J, Cavarretta I, Pozzi E, Lavorgna G, Locatelli I, Tempio T, Montorsi F, Mattei A, Sitia R, Salonia A, Anelli T. Endoplasmic reticulum oxidoreductase 1 alpha modulates prostate cancer hallmarks. Transl Androl Urol 2021;10(3):1110-1120. doi: 10.21037/tau-20-1025 through modulation of integrin-beta 1 modification and signalling in HCT116 colorectal cancer cells. Sci Rep 2017;7:9389.

41. Hayes KE, Batsomboon P, Chen W-C, et al. Inhibition of the FAD containing ER oxidoreductin 1 (Ero1) protein by EN-460 as a strategy for treatment of multiple myeloma. Bioorg Med Chem 2019;27:1479-88.

42. Hynes RO. Integrins: versatility, modulation, and signaling in cell adhesion. Cell 1992;69:11-25.

43. Pan B, Guo J, Liao Q, et al. beta 1 and beta 3 integrins in breast, prostate and pancreatic cancer: A novel implication. Oncol Lett 2018;15:5412-6.

44. Fay AP, Antonarakis ES. Blocking the PD-1/PD-L1 axis in advanced prostate cancer: are we moving in the right direction? Ann Transl Med 2019;7:S7.

45. Li H, Wang Z, Zhang Y, et al. The Immune Checkpoint Regulator PDL1 is an Independent Prognostic Biomarker for Biochemical Recurrence in Prostate Cancer Patients Following Adjuvant Hormonal Therapy. J Cancer 2019;10:3102-11.

46. Melegh Z, Oltean S. Targeting Angiogenesis in Prostate Cancer. Int J Mol Sci 2019;20:2676.

47. Abdulghani J, Gu L, Dagvadorj A, et al. Stat3 promotes metastatic progression of prostate cancer. Am J Pathol 2008;172:1717-28. 